\title{
The Effects of Scientific Reasoning Skills, English Language Proficiency and other Factors on the Acquisition of Chemistry Content Knowledge
}

\author{
Manolito G. Ybañez, Jr, Charmaine Sagayap, Vic Marie I. Camacho* \\ Philippine Normal University, Taft Avenue, Manila 1000
}

\begin{abstract}
This paper aims to examine the effects of English Language Proficiency, Scientific Reasoning Skills, and other factors such as primary language at home, subject interests, type of school graduated, family monthly income, and available studying materials on acquisition of Chemistry content knowledge of fifty-four (54) randomly selected preservice science teachers from a state university. Using One-way Analysis of Variance (ANOVA) and Pearson $\mathrm{r}$ formula, the results of this 2 -way interaction showed an $\mathrm{F}$ ratio of 1.025 with a significance level of 0.445 . This finding is significant as it implies that combined high levels of English language proficiency and reasoning skills enhance students' abilities to learn science content. In addition, a significant main effect for the variable scientific reasoning skills, an $\mathrm{F}$ ratio of 0.550 with a significance level of 0.815 show that English Proficiency does not significantly affect the Chemistry Skills of the Science Students. Science Reasoning Skills is correlated with Chemistry performance. Further, higher scientific reasoning skills are attributed to better Chemistry Skills. Primary language significantly affects language proficiency while subject interest affect the students skills in subject areas.
\end{abstract}

Keywords: Scientific reasoning skills; English language proficiency; acquisition of chemistry content knowledge; Undergraduate Science majors

\section{INTRODUCTION}

Scientific literacy for all students has been a major educational goal in the Philippines. The major educational $\mathrm{K}$ to 12 reform stresses the need for a prepared 21st century workforce, which aimed not only of students' content learning but also in acquiring transferable reasoning skills. By understanding science and applying scientific skills, personal decisions on issues that affect society as well as in reporting scientific discovery and comprehension can be done using scientific processes. Often, this understanding of science has proven to be very elusive for Filipino language learners. As stated by the American Association for the Advancement of Science, neglecting the science education of students is to deprive them of a basic education, handicap them for life, and deprive the nation of talented workers and informed citizens.

In most classrooms in the Philippines, the English language is used as the medium of 
instruction for science (Florido, 2006). Therefore, one of the objectives of this research study was to examine the effects of English language proficiency on the acquisition of Chemistry content knowledge by Filipino English language learners. The theoretical foundation to investigate this factor was Cummins' work as explained by Haynes (1998) on cognitive academic language proficiency, which relates both cognitive and linguistic processes to the academic success of students, more specifically non-native English language learners. Cummins proposed two levels of language proficiency: the basic interpersonal communicative skills (BICS) and the cognitive academic language proficiency (CALP). The basic interpersonal communicative skills (BICS) concept represents the language of natural, informal conversation. Basic interpersonal communicative skills (BICS) are used by students when talking about everyday things in concrete situations, that is, situations in which the context provides cues that make understanding not totally dependent on verbal interaction alone. Cummins refers to this everyday conversational ability as context embedded or contextualized. It has been found that in context embedded or contextualized communication, the conversation deals with familiar events or matters that require that the speakers react and respond to each other. However, CALP is the type of language proficiency needed to read textbooks, to participate in dialogue and debate, and to provide written responses to tests. Students who have not yet developed their cognitiveacademic language proficiency (CALP) could be, according to these researchers, at a disadvantage in learning science or other academic subject matter.

To measure CALP, the researchers used the Test of English as a Second Language (TOEFL). TOEFL measures the ability of non-native speakers to use for the purposes of learning in higher education institutions. TOEFL, a worldwide recognized language test entrance qualification for a variety of international degree programs all over the world. This language test, however in this particular study, is utilized to measure BICS.
Figure 1 shows that above the surface of the water line, the cognitive process includes knowledge, comprehension and application; and language process includes pronunciation, vocabulary and grammar. In this representation, basic interpersonal communications skills (BICS), or skills, which depend on the surface features of language and lower levels of cognitive processes, are represented above the waterline CALP or skills related to the meaning of language and higher level of cognitive processes are represented below the waterline. Thus, the test is limited to measure CALP.

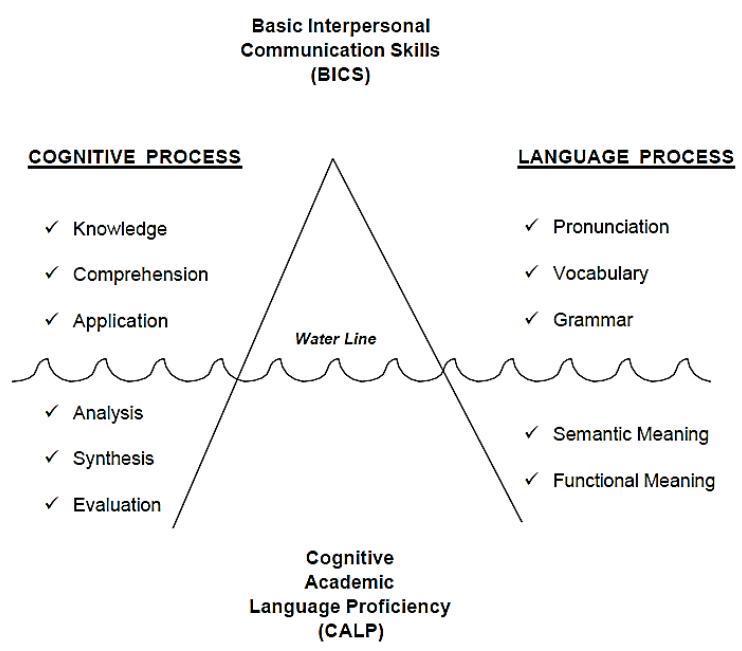

Figure 1. Surface and deeper levels of language proficiency (Rosenthal, 1996).

A second objective of this study was to investigate the effects of scientific reasoning skills on the acquisition of Chemistry content knowledge by Filipino students participating in second year college classes. There are several instruments that measure scientific reasoning skills such as the Lawson's Test of Scientific Reasoning (LTSR) v 2000, the inventory for scientific thinking and Reasoning (iSTAR) $\mathrm{v}$ 2011 to mention a few. To examine this factor, the researchers drew on the work of Lawson, et al. on levels of scientific reasoning skills, because it provides a starting point of assessing scientific reasoning and it measures skills that concretely the broadly defined scientific reasoning abilities. A study utilized the Lawson's Test for Classroom Scientific Reasoning to Chinese and U.S. suggest that there is no variation in students' scientificreasoning abilities despite the large differences 
in $\mathrm{K}-12$ STEM education between the United States and China (Bao and Koenig, 2014). The use of a general pattern of formal reasoning is necessary for the acquisition of new science concepts that is, during the formal operational stage where thinking reaches its highest degree of equilibrium, students acquire scientific thinking with its hypothetico-deductive reasoning and logical reasoning with its interpropositional reasoning. This corroborates the statement of Piaget, that the various operations are tightly interrelated and that they apply to the widest possible field of application, that is, the realm of hypothetical possibilities. In this study, CALP will be measured using the scientific reasoning test. The National Competitive Examination (NCE), a standardized qualifying examination in the country was administered to measure Scientific Reasoning Skills. The 30-item NCE consists of three sections: Mathematics, Science and Abstract Reasoning usually administered to qualifiers for Science, Technology, Engineering and Mathematics courses.

There are relatively few studies examining the effects of both English language proficiency and Scientific reasoning skills on the learning of Scientific content knowledge of students. Both past research and theory suggested that English language proficiency and levels of scientific reasoning skills could affect or are prerequisite for the acquisition of science content knowledge as reported by a study conducted by Zeidler and Torres (2002) to Grade 10 Hispanic learners. Accordingly, the researchers choose to further investigate and delineate this presupposition this time, applying in Filipino students.

Filipino Language Learners. English language proficiency and levels of scientific reasoning skills of Filipino language learners are two variables on their acquisition of science content knowledge are to be examined. Preservice science teachers taking General Chemistry 2 classes were deemed appropriate and relevant respondents, for at this age, students display a variety of reasoning abilities but are often called upon to learn concepts which require the use of formal reasoning skills.
Piaget documented that the process of scientific reasoning becomes formal operational during adolescence. There is evidence which suggests that individuals have the ability to use their formal reasoning skills somewhat more consistently at the second year college level (18 years old) than earlier year in which students are apt to be transitional (Balce, 2010).

Science Skills. Science is frequently perceived to be of great importance because of its links to technology and industry which, from a national perspective, may be areas with high priority for development. Consequently, science is considered a fundamental element in elementary and secondary preparations despite conceptual complexity. Another justification for the inclusion of science in school curricula is that all citizens need to achieve a degree of "scientific literacy" to enable them to participate effectively as citizens in modern societies (Batomalaque, 2003). Studies indicate however, that many of our Filipino learners are not attaining functional literacy, without which they find it too difficult to meet the challenges posed by our rapidly changing world. Students' performance in National Achievement Tests shows that aside from Mathematics, Science continues to be the most difficult field of study in basic education. As for the 1999-2000 National Elementary Achievement Test (NEAT), pupils gave correct answers to less than $50 \%$ of the questions in Science (48.61\%) and mathematics (45.69\%). The main factors, which can be cited to account for the low performance in science of the Filipino student, include the lack of science culture and deficiencies regarding the school curriculum, the teaching learning process, instructional materials and teacher training. Studies regarding the effect of language proficiency in the acquisition of knowledge was not established.

English Language Proficiency. Language is an integral part of culture, and the words that we have and how we use them reflect our values and belief system (Rosenthal, 1996). The native language we speak and use is determined by the culture in which we are raised and schooled. There is a distinction between unconscious language acquisition and conscious language 
learning. Unconscious language acquisition is attributed to the native language proficiency, which everyone develops, while conscious language learning refers to studying a second language taught by teachers, using textbooks, taking formal classes and learning the rules, vocabulary, grammar, and idioms of the second language. In the case of Filipino language learners, students may become quite proficient in the grammar, vocabulary and sentence structure of the English language, but may lack the necessary cognitive academic language proficiency to learn the subject matter that is presented to them in science classrooms. In other words, these students may be proficient in their English communication skills but may not have the cognitive academic language proficiency (CALP) required for learning science or other academic subject matter. Cummins contends that all children develop basic interpersonal communicative skills (BICS) and learn to communicate in their native or first language and that cognitive academic language proficiency (CALP) reflects a combination of language proficiency and cognitive processes that determines a student's success in school.

Other Factors. To better understand the preservice science teachers acquisition of content knowledge, other factors such as primary Language at home, subject interests, type of school graduated, family monthly income, and available studying materials were considered important to be included in this study.

In this study, the following research questions were investigated: 1.) Do monthly family income, subject interest, primary language use, type of school graduated in high school and types of studying materials at home affect the acquisition of Chemistry content knowledge? 2.) What are the effects of English language proficiency and levels of Scientific reasoning skills on the acquisition of Chemistry content knowledge of Filipino language learners participating in second year college science classes?

\section{METHODOLOGY}

Despite the vast number of studies on factors influencing the academic performance of
Filipino students, investigation of the effects of two very important factors, English language proficiency and scientific reasoning skills, on the acquisition of Chemistry content knowledge of Filipino language learners is a relatively neglected research area. Prior to the main question of this study, the researchers have examined the effects of monthly family income, subject interest, primary language use, type of school graduated in high school and types of studying materials at home. This research study examined these two factors, English language proficiency and scientific reasoning skills, and provides some empirical evidence for addressing the research questions posted above.

The subject pool identified for the study consisted of 54 pre-service science teachers who majors in Chemistry, General Sciences, and Physics Teaching and considered to be non-native English speakers from the National Center for Teacher Education, the Philippine Normal University. The random selection of the students was based on science majors because these fields of science utilize scientific, mathematical, and language reasoning. The respondents were composed of 34 females and 20 males. $96 \%$ of the respondents use Filipino as their primary language at home, $31 \%$ have a monthly family income of less than 10,000 PhP; $19 \%$ for more than $20,000 \mathrm{PhP} ; 22 \%$ for 10,000 to $15,000 \mathrm{PhP}$; and $28 \%$ for 15,000 to 20,000 $\mathrm{PhP}$.

Instruments. The demographic profile of the respondents was prepared which specifically includes the monthly family income, subject interest, primary language use, type of school graduated in high school and types of studying materials at home.

Test of English as a Foreign Language (TOEFL) instrument, developed by Educational Testing Service (ETS) is a 30item test used to determine the language proficiency of the students in this study (See supporting Information). The test is composed entirely of multiple-choice questions with four possible answers per question. There are three sections in the test, 
each measuring a critical skill in the use of English namely, listening comprehension, structure and written expression and reading comprehension (Princeton, 2004). In the assessment of the students' performance on the test, responses were categorized into five (5) levels of English language proficiency is designated as: poor, below average, average, above average and excellent as adopted from the English Language Proficiency Levels. The classification was based on the following numerical range:

Table 1: TOEFL Interpretation.

\begin{tabular}{lc}
\hline Level & Range \\
\hline Poor & $1-14$ \\
\hline Below Average & $15-19$ \\
\hline Average & $20-24$ \\
\hline Above Average & $25-27$ \\
\hline Excellent & $28-30$ \\
\hline
\end{tabular}

The National Competitive Examination (NCE), a standardized qualifying examination was administered to respondents to measure Scientific Reasoning Skills. The 30-item NCE consists of three sections: Mathematics, which measures how well the students understand the number system and the terms and operations used in Mathematics; Science, which measures the performance and natural ability in science; and Abstract Reasoning, which measures the ability to recognize logical patterns from non-verbal cues or images and their relationships, and to make predictions on these patterns. NCE scores were interpreted using the classification used in TOEFL as shown in Table 1.

The results of the test were analyzed and correlated with the results of Chemistry Departmental Examination of the students a week after TOEFL and Scientific Reasoning Test were administered.

The statistical technique of Pearson $r$ test of correlation and ANOVA test of variance were applied to determine the said correlation.

\section{RESULTS AND DISCUSSION}

A $3 \times 3$ between-subjects factorial design was employed to evaluate simultaneously the effect of the two independent variables, i.e., English language proficiency, and scientific reasoning) on the dependent variable, i.e., chemistry content knowledge. The English language proficiency comprised three categories, i.e. listening comprehension, structure and written expressions, and reading comprehension, as did the scientific reasoning variable, i.e., mathematics, science, and abstract reasoning. One-way analysis of variance (ANOVA) was used to evaluate there was an interaction among the two independent variables on the chemistry achievement of science majors.

Respondents' Profile. Descriptive data and frequency histograms for the factors such as primary Language at home, subject interests, type of school graduated, family monthly income, and available studying materials are discussed below.

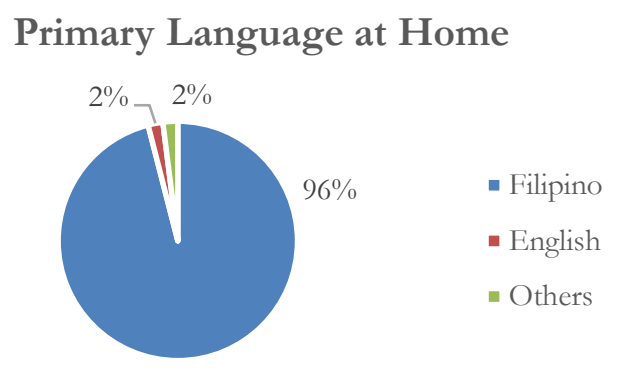

Figure 2. The Primary Language use at Home.

Figure 2 shows that most of the respondents use Filipino as their primary language at home. In comparison to the variables, the primary language affects the English language proficiency. In general, those students who said that English is their primary language at home did well in Language test; those students who use Filipino as their home language performed average; and those students who use other languages (or dialects) perform not so well in language test. This data however does not affect the Chemistry performance. 


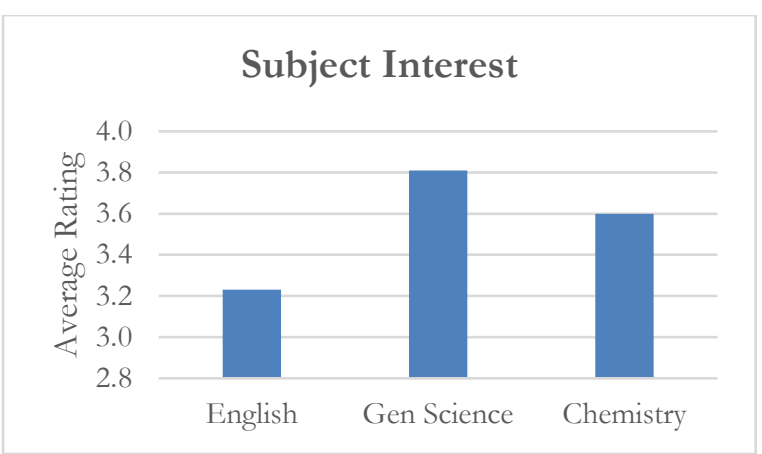

Figure 3. The Respondents' Subject Interest.

Figure 3 gives a view of the students' subject interests. Most of the students placed Science in general as a subject that interested them the most. This has correlated to higher scientific reasoning skills of the respondents. Those who placed English as their primary interest did well in Language test; those who like Chemistry also performed well in the Chemistry and scientific test.

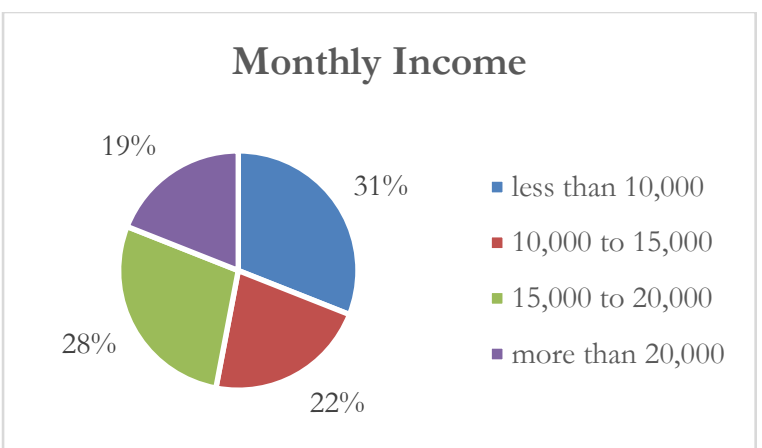

Figure 4. The Respondents' Monthly Family Income.

The data in figure 4 shows no significance to any of the variable studied. The family monthly income does not affect the respondents' ability in English, Science and Chemistry.

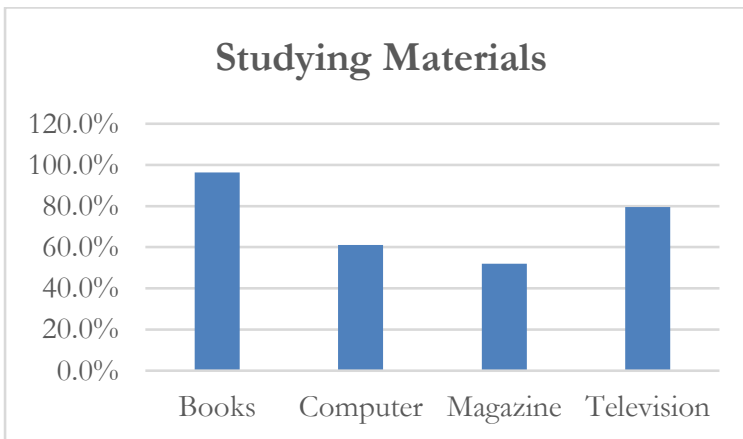

Figure 5. The Respondents' Studying Materials at Home.
This data in Figure 5 also shows no significance on the students' performance in different variables. Thus, the study materials available at home do not affect their ability in English, Science and Chemistry.

\section{Type of School Graduated}

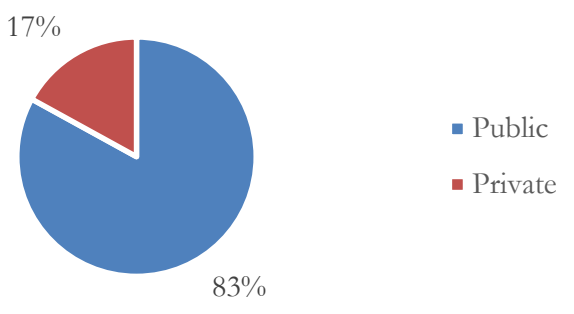

Figure 6. The Respondents' Type of School Graduated in HS.

This data (Figure 6) also shows no significance on the students' performance in different variables. Thus, the type of graduated in high school does not affect their ability in English, Science and Chemistry. This could be attributed to the highly selective admission process for the pre-service science students.

English Language Proficiency and Chemistry Performance. Figure 7 shows the various categories of English language proficiency. For English language proficiency, one (1) student were categorized as having poor English language proficiency, two (2) as having below average, twenty-four (24) as having average, seventeen (17) as having above average and nine (9) as having excellent proficiency.

\section{English Language Proficiency Distribution}

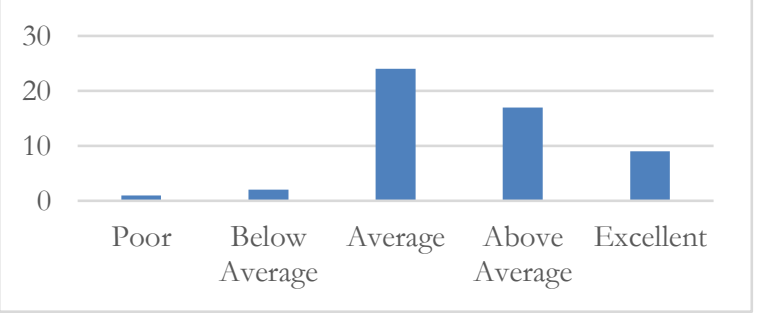

Figures 7. Frequency Distribution for English Language Proficiency Levels as Measured by TOEFL $(N=54)$. 
The results of the one-way analysis of variance presented in Table 2 showed that an $F$ ratio of 1.025 is greater than the significance of .445 for the interaction of Levels of English Language Proficiency with regard to the students' performance on the standardized chemistry test.

Table 2. One-way Analysis of Variance $(\mathbf{N}=54)$.

\section{ANOVA}

Language Proficiency vs. Chemistry

Performance

\begin{tabular}{lccccc}
\hline & $\begin{array}{c}\text { Sum of } \\
\text { Squares }\end{array}$ & df & $\begin{array}{c}\text { Mean } \\
\text { Square }\end{array}$ & F & Sig. \\
\hline $\begin{array}{l}\text { Between } \\
\text { Groups }\end{array}$ & 1025.351 & 12 & 85.446 & $\mathbf{1 . 0 2 5}$ & $\mathbf{. 4 4 5}$ \\
\hline $\begin{array}{l}\text { Within } \\
\text { Groups }\end{array}$ & 3417.482 & 41 & 83.353 & & \\
\hline Total & $\mathbf{4 4 4 2 . 8 3 3}$ & $\mathbf{5 3}$ & & & \\
\hline
\end{tabular}

Analysis of Variance (ANOVA) for Hypothesis H1AO. The hypotheses developed and examined for this study are related to Cummins' theoretical framework on cognitive academic language proficiency. As previously noted, the researchers evaluated the hypotheses using a between-subjects factorial analysis of variance (ANOVA). The following hypotheses were developed to examine Cummins' claim on the need for students to have cognitive academic language proficiency as a prerequisite to learning Chemistry content subject matter:

H10: There is no significant difference between the result of English language proficiency and Chemistry content knowledge acquisition. That is, the students who will get high score in TOEFL will have a high score in Chemistry test.

$\mathrm{H} 1_{\mathrm{a}}$ : There is a significant difference between the result of English language proficiency and Chemistry content knowledge acquisition. That is, the students' score in TOEFL will not be the same with Chemistry test.

The ANOVA technique tested the null hypothesis (H10) that the sample means or students' average scores on the standardized science test were equal. The results of the analysis of variance (ANOVA) are shown in Table 3. Table 3 shows a ratio of means squares $(F)$ of 1.025 and a significance level (Sig) of .445; that is; the significance value is lesser than the $F$ value. Hence, the null hypothesis (H10) was rejected for it was unlikely that the average scores on the Chemistry test were not the same for the group of science major students with 5 different levels of English language proficiency.

\section{Science Reasoning Skills and Chemistry} Performance. Figure 8 shows the number of students in various categories of Science Reasoning. Four (4) student were categorized as having poor science reasoning skills, seven (7) as having below average, twenty (20) as having average, sixteen (16) as having above average and five (5) as having excellent proficiency.

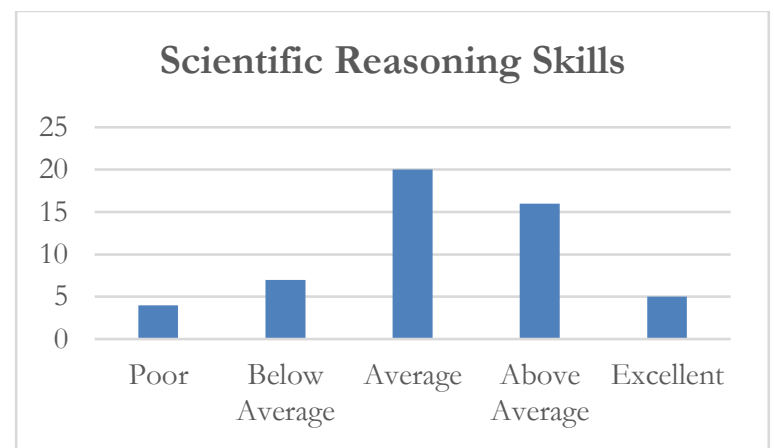

Figure 8: Frequency Distribution for Scientific Reasoning Skill Levels as Measured by Lawson's Instrument $(\mathrm{N}=54)$.

For the ANOVA, the researchers first tested the interaction between two of the variables. The results of the one-way analysis of variance are shown in Table 3 below. The results of the one-way analysis of variance showed significant 2-way interaction between the variables in the study. Table 3 shows an $F$ ratio of 0.550 and with a significance of .815 for the interaction of Levels of science reasoning skills with regard to the students' performance on the standardized chemistry test. 
Table 3: One-way Analysis of Variance $(\mathbf{N}=54)$.

Scientific Reasoning Skills versus Chemistry Performance

\begin{tabular}{llllll}
\hline & $\begin{array}{l}\text { Sum of } \\
\text { Squares }\end{array}$ & df & $\begin{array}{l}\text { Mean } \\
\text { Square }\end{array}$ & F & Sig. \\
\hline $\begin{array}{l}\text { Between } \\
\text { Groups }\end{array}$ & 391.873 & 10 & 39.187 & $\mathbf{. 5 5 0}$ & $\mathbf{. 8 1 5}$ \\
\hline $\begin{array}{l}\text { Within } \\
\text { Groups }\end{array}$ & 569.917 & 8 & 71.240 & & \\
\hline Total & $\mathbf{9 6 1 . 7 8 9}$ & $\mathbf{1 8}$ & & & \\
\hline
\end{tabular}

The hypotheses developed and examined for this area of study are related to Lawson et al. research studies on scientific reasoning skills. As previously noted, the researchers evaluated the hypotheses using a between-subjects factorial analysis of variance (ANOVA). The following hypotheses were developed to examine Lawson's claim on the need for students to have science reasoning skills as a prerequisite to learning Chemistry content subject matter:

H20: There is no significant difference between the result of Scientific Reasoning Test and Chemistry content knowledge acquisition. That is, the students who will get high score in Scientific Reasoning Test will have a high score in Chemistry test.

$\mathrm{H} 2$ : There is significant difference between the result of Scientific Reasoning Test and Chemistry content knowledge acquisition. That is, the students' score in Scientific Reasoning Test will not be the same with Chemistry test.

The researchers once again, used an analysis of variance (ANOVA) technique to test the null hypothesis H20. The ANOVA technique tested the null hypothesis (H20) that the average test scores on the standardized science test for the groups were equal. The results of the analysis of variance (ANOVA) are shown in Table 4. Table 4 shows a ratio of means squares $(F)$ of 0.550 and a significance level (Sig) of .815; which means that the significance level is greater than the $F$ value. Hence, the null hypothesis (H20) was accepted for it is unlikely that the average scores on the Chemistry test were the same for the group of science major students with 5 different levels of English language proficiency.

Tables 3 and 4 showed the 2-way interaction between the English Language proficiency and scientific reasoning skills variables with regard to the students' performance on the standardized chemistry test. The results of this 2-way interaction showed an $F$ ratio of 1.025 with a significant level of .445 . This finding is believed to be important for it implies that combined high levels of English language proficiency and reasoning skills enhance students' abilities to learn science content subject matter. In addition, Table 4 showed a significant main effect for the variable reasoning skills (i.e., an $F$ ratio of 0.550 with a significant level of .815). No significant main effect was shown in Table 3 for English language proficiency and Chemistry skills.

It was also found out that the primary language used at home affects the language proficiency of the students. It positively correlates with the results of the TOEFL. The subject interest draws another effect on their scientific reasoning skills.

\section{CONCLUSIONS}

In light of the findings of this undertaking, following points were concluded: (1) The English language proficiency is not a reliable indicator of competency in Chemistry content knowledge. It is found out that having a high score in a language test does not guarantee a high score in Chemistry exams. Thus, English language proficiency does not affect Chemistry content knowledge. (2) The Scientific Reasoning Skills positively correlates with Chemistry performance. It is observed that Scientific Reasoning skills do affect the students' performance in Chemistry. On the other data, it is examined that the primary language used at home affects the English proficiency of the students. The subject interest somehow affects the performance of the students in each subject. The monthly income and type of school graduated does not 
affect the English proficiency, Science reasoning skills and Chemistry skills. (3) Only the primary language used at home and subject interest affect the students' competency in every variable measure.

\section{IMPLICATIONS TO CHEMISTRY TEACHING}

This study also draws the following implications in chemistry teaching: (1) The students' English Language Skills might be very helpful to the students but it does not necessarily dictate the student's skills in different aspects. (2) In order to teach science effectively, teachers must develop the students' scientific reasoning skills. This might be a pre-requisite to chemistry education. (3) Factors such as interest and language use affect the skills of a student. Other factors (i.e., monthly family income, type of school graduated and available studying materials at home) were found to be not contributory to the skills of the student. (4) The monthly family income, type of school graduated in high school and studying materials at home do not affect their performance in different variables.

The validity and reliability of the results can be increased increasing the number of respondents. It is recommended that this study be replicated using a greater number of respondents in varied school environments to further support the results.

The test items in an assessment provide and create the color of the interpretation. Here, some of the test items used might have been so easy and some might be so hard. It is so advisable to use a table of specifications to locate and plan the coverage of the test.

The researcher may recommend further studies about Language and Science relationship with regard to other factors. Other researchers may use different material and tools to provide more meaningful and valid data.

\section{REFERENCES}

Balce M. Teaching Quality Science Education in Filipino [Internet]. Quezon City, Philippines: University of the Philippines; 2010. Available from: https://mlephil.wordpress.com/2010/02/26/ teaching-quality-science-education-infilipino/.

Bao and Koenig. What is Scientific Reasoning and Why is It Important? [nternet]. Place unknown]: International Partnership of Education Research and Communication; 2014 [cited 2016 Aug 24]. Available from: http://www.iperc.org/home/research-sr/.

Batomalaque A. Basic Science Development Program of the Philippines for International Cooperation. Cebu City, Philippines: University of San Carlos; 2003.

Cummins J. Concepts of BICS and CALP. Basic Interpersonal Communities Skills and Cognitive Academic Language Proficiency. Toronto, Canada: University of Toronto; 2009.

Haynes J. Explaining BICS and CALP [Internet]. [Place unknown]: [Publisher unknown]; 1998 [cited 2012 Mar 10]. Available from: http://www.everythingesl.net/inservices/bics _calp.php.

Florido A. Educational Profile of the Philippines [Internet]. [Place unknown]: Charlotte Mecklenburg Schools; 2006. Available from: http://www.unc.edu/world/2006_K12Symp/ Pres\&HOs/Florido_Handout1.pdf.

Lawson AE. The development and validation of a classroom test of formal reasoning. J Res Sci Teach. 1978; 15(1) 11-24. In: Classroom Test of Scientific Reasoning. Revised ed. 2000.

Learning Language Proficiency Levels [Internet]. [Place unknown]: [Publisher unknown]; 2012 [cited 2012 Mar 10]. Available from: https://ells.wiki.farmington. k12.mi.us/ELL+Proficiency+Levels. 
Sample test items in English Language Proficiency. Test of English as a Foreign Language Institutional Testing Program (TOEFL Testing Program). Princeton, NJ; [Publisher unknown]; 2004.

Rosenthal JW. Teaching Science to Language Minority Students. Multilingual Matters LTD. [Place unknown]: Taylor \& Francis; 1996. 48 p.

Test of English as a Foreign Language (TOEFL). [Place unknown]: Internet Course Finders; 2012.
Torres and Zeidler. The Effects of English Language Proficiency and Scientific Reasoning Skills on the Acquisition of Science Content Knowledge by Hispanic English Language Learners and Native English Language Speaking Students. Electron J Sci Educ. 2002; 6(3). Available from: http://ejse.southwestern.edu/issue/view/730. 